\title{
On the Capacity Regions of Single-Channel and Multi-Channel Full-Duplex Links
}

\author{
Jelena Marašević and Gil Zussman \\ Department of Electrical Engineering, \\ Columbia University \\ New York, NY, 10027, USA \\ \{jelena, gil\}@ee.columbia.edu
}

\begin{abstract}
We study the achievable capacity regions of full-duplex links in the single- and multi-channel cases (in the latter case, the channels are assumed to be orthogonal - e.g., OFDM). We present analytical results that characterize the uplink and downlink capacity region and efficient algorithms for computing rate pairs at the region's boundary. We also provide near-optimal and heuristic algorithms that "convexify" the capacity region when it is not convex. The convexified region corresponds to a combination of a few full-duplex rates (i.e., to time sharing between different operation modes). The algorithms can be used for theoretical characterization of the capacity region as well as for resource (time, power, and channel) allocation with the objective of maximizing the sum of the rates when one of them (uplink or downlink) must be guaranteed (e.g., due to QoS considerations). We numerically illustrate the capacity regions and the rate gains (compared to time division duplex) for various channel and cancellation scenarios. The analytical results provide insights into the properties of the full-duplex capacity region and are essential for future development of scheduling, channel allocation, and power control algorithms.
\end{abstract}

\section{Categories and Subject Descriptors}

C.2.1 [Computer-Communication Networks]: Network Architecture and Design-Wireless Communication

\section{Keywords}

Full-Duplex; Resource Allocation; Capacity Region

\section{INTRODUCTION}

Existing wireless systems are Half-Duplex (HD), where separating the transmitted and received signal in either frequency or time causes inefficient utilization of the wireless resources. An emerging technology that can substantially improve spectrum efficiency is Full-Duplex (FD) wireless, namely, simultaneous transmission and reception on the same

Permission to make digital or hard copies of all or part of this work for personal or classroom use is granted without fee provided that copies are not made or distributed for profit or commercial advantage and that copies bear this notice and the full citation on the first page. Copyrights for components of this work owned by others than the author(s) must be honored. Abstracting with credit is permitted. To copy otherwise, or republish, to post on servers or to redistribute to lists, requires prior specific permission and/or a fee. Request permissions from permissions@acm.org.

MobiHoc'16, July 04 - 08, 2016, Paderborn, Germany

(c) 2016 Copyright held by the owner/author(s). Publication rights licensed to ACM ISBN 978-1-4503-4184-4/16/07 . \$ \$15.00

DOI: http://dx.doi.org/10.1145/2942358.2942383

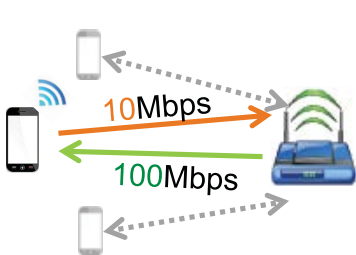

(a)

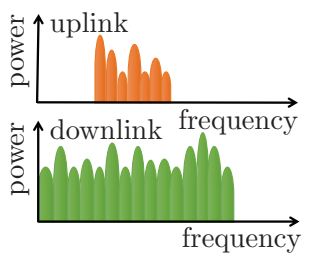

(c)

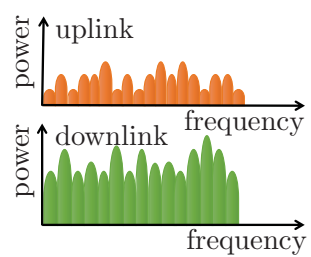

(b)

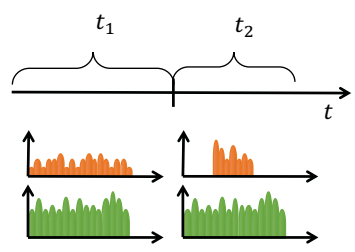

(d)
Figure 1: (a) An example of different rate requirements on a full-duplex link and possible policies to meet the requirements: (b) reduction of the the power levels on the UL channels, (c) allocation of a subset of the channels to the UL, and (d) time-sharing between two FD rate pairs (TDFD).

frequency channel [20]. The main challenge in implementing FD devices is the high Self-Interference (SI) caused by signal leakage from the transmitter into the receiver. The SI signal is usually many orders of magnitude higher than the desired signal at the receiver's input, requiring over $100 \mathrm{~dB}\left(10^{10}\right.$ times) of Self-Interference Cancellation (SIC). Recently, several groups demonstrated that combining techniques in the analog and digital domains can provide SIC that can support practical applications (e.g., $[2,5,8,10,13,14,24])$.

The first implementations of FD receivers optimistically envisioned $2 \times$ data rate improvement (e.g., [5, 13]). However, such a rate increase requires perfect SIC, which is extremely challenging to achieve. While a few recent papers considered non-negligible SI and the resulting rate gains $[1,7$, $16,17]$, there is still no explicit characterization of the FD capacity region for a given profile of residual SI over frequency ${ }^{1}$ and parameters of the wireless signal. Most recent research has focused on maximizing the total throughput without considering Quality of Service (QoS) requirements. Namely, there has been very limited work on asymmetric traffic requirements on the uplink (UL) and downlink (DL) [6,15-17].

While in Time Division Duplex (TDD) systems asymmet-

\footnotetext{
${ }^{1}$ In compact FD radio implementations (e.g., [24]), the residual SI can vary wildly with the frequency.
} 
ric traffic can be supported via time-sharing between the UL and DL, in FD the dependence of the bi-directional rates on the transmission power levels and Signal-to-Noise Ratio (SNR) levels is much more complex. As shown in Fig. 1, any (combination) of the following policies can be used: (i) FD with reduced transmission power at one of the stations, (ii) FD with fewer channels allocated to one of the stations, and (iii) time sharing between a few types of FD transmissions.

We study asymmetric link traffic and analytically characterize the capacity region (i.e., all possible combinations of UL and DL rates) under non-negligible SI. Such characterization has theoretical importance, since it provides insights into the achievable gains from FD, thereby allowing to quantify the benefits in relation to the costs (in hardware and algorithmic complexity, power consumption, etc.). It also has practical importance, since it supports the development of algorithms for rate allocation under different UL and DL requirements. Such algorithms will determine the required combinations of the policies illustrated in Fig. 1.

We first consider the case where both stations transmit on a single channel and the remaining SI is a constant fraction of the transmitted power $[6,17]$. We study the structural properties of the FD capacity region and derive necessary and sufficient conditions for its convexity. Based on the properties, we present a simple and fast algorithm to "convexify" the region. $^{2}$ The convexified region combines (via time sharing) different FD rate pairs (see Fig. 1(d)) and we refer to it as the Time Division Full-Duplex (TDFD) region. The algorithm finds the points at the region's boundary, given a constraint on one of the (UL or DL) rates.

We then consider the the multi-channel case in which channels are orthogonal, as in Orthogonal Frequency Division Multiplexing (OFDM). We assume that the shape of the power allocation is fixed but the total transmission power can be varied. For each channel, the remaining SI is some fraction of the transmitted power $[7,17,23]$. We characterize the FD capacity region and analytically show that any point on the region can be computed with a low-complexity binary search. We also focus on determining the TDFD capacity region, which due to the lack of structure cannot in general be obtained via binary search. However, we argue that for any practical input, the TDFD capacity region can be determined in real time.

Finally, we consider the TDFD capacity region in the multi-channel case under a general power allocation, (i.e., the power level at each channel is a decision variable). In this case, maximizing one of the rates when the other rate is given is a non-convex problem which is hard to solve. However, we develop an algorithm that under certain mild restrictions converges to a stationary point that in practice is a global maximum. Although for most practical cases, the algorithm is near-optimal and runs in polynomial time, its running time is not suitable for a real-time implementation. Hence, we develop a simple heuristic and show numerically that in most cases it has similar performance.

For all the cases mentioned above, we present extensive numerical results that illustrate the capacity regions and the rate gains (compared to TDD) as a function of the receivers' SNR levels and SIC levels. We also highlight the intuition behind the performance of the different algorithms.

\footnotetext{
${ }^{2} \mathrm{~A}$ convex region is desirable, since most resource allocation and scheduling algorithms rely on convexity and providing performance guarantees for a non-convex region is hard.
}

To summarize, the main contributions of the paper are two-fold: (i) it provides a fundamental characterization and structural understanding of the FD capacity regions, and (ii) the rate maximization algorithms, designed for asymmetrical traffic requirements, can serve as resource allocation building blocks for future FD MAC protocols.

The rest of the paper is organized as follows. Sections 2 and 3 review related work and outline the model. Section 4 studies the single channel case. Sections 5 and 6 study the multi-channel cases with fixed and general power allocations. We conclude in Section 7. Due to space constraints, some of the proofs are omitted and appear in a technical report [18].

\section{RELATED WORK}

Various challenges related to FD wireless recently attracted significant attention. These include FD radio/system design $[2,5,8,13,14,24]$ as well as rate gain evaluation and resource allocation $[1,3,6,7,16,17,21-23]$. A large body of (analytical) work $[3,21,22]$ focuses on perfect SIC while we focus on the more realistic model of imperfect SIC.

Rate gains and power allocation under imperfect SIC were studied in $[1,6,7,16,17,23]$. For the single channel case, [1] derives a sufficient condition for FD to outperform TDD in terms of sum UL and DL rates. However, [1] does not quantify the rate gains nor consider the multi-channel case.

Power allocation for maximizing the sum of the UL and DL rates for the single- and multi-channel cases was studied in $[7,17]$. The maximization only determines a single point on the capacity region and does not imply anything about the rest of the region, which is our focus. While [17] (implicitly) constructs the FD capacity region in the single channel case (restated here as Proposition 4.1), it does not derive any structural properties of the region, nor does it consider the multi-channel case or a combination of FD and TDD.

The capacity region for an FD MIMO two-way relay channel was studied in [23] as a joint problem of beamforming and power allocation. For a fixed beamforming, the problem reduces to determining a single channel FD capacity region. Yet, the joint problem is significantly different from the problems considered here. The FD capacity region for multiple channels was considered in [16]. While [16] considers both fixed and general power allocation for determining an FD capacity region, the analytical results are obtained only for the fixed power case and the non-convex problem of general power allocation was addressed heuristically. Specifically, for the fixed power case, our proof of Lemma 5.1 is more accurate than the proof of Theorem 3 in [16] (see [18]).

The TDFD capacity region was studied in [15] only via simulation and in [6] analytically but mainly for the singlechannel case. The "convexification" of the FD region in [6] is performed over a discrete set of rate pairs, which requires linear computation in the set size, assuming that the points are sorted (e.g., Ch. 33 in [9]). Our results for a single channel rely on the structural properties of the FD capacity region and do not require the set of $\mathrm{FD}$ rate pairs to be discrete. Moreover, the computation for determining the convexified region is logarithmic (see Section 4.2).

To the best of our knowledge, this is the first thorough study of the capacity region and rate gains of FD and TDFD.

\section{MODEL AND NOTATION}

We focus on the problem of determining the capacity re- 


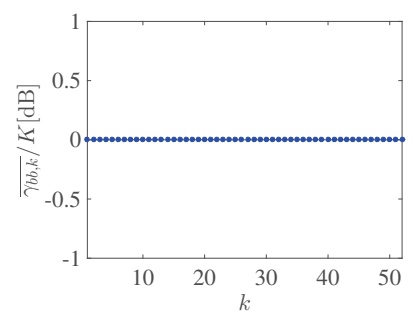

(a)

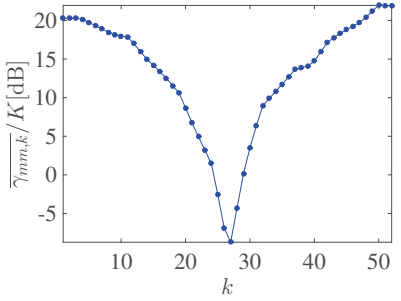

(b)

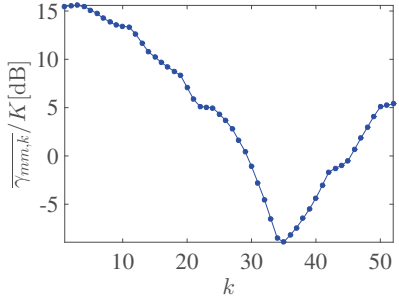

(c)

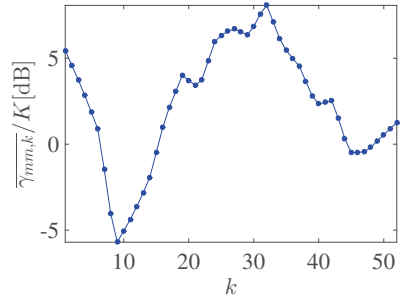

(d)

Figure 2: Considered cancellation profiles for the FD receiver (a) at the BS [5] and (b), (c), (d) at the MS [24].

gion of an FD bidirectional link between two stations. For brevity, we refer to them as a Mobile Station (MS) and a Base Station (BS) and to the corresponding links as uplink (UL) and downlink (DL). For the number of channels $K$, we consider: (i) the single-channel case $(K=1)$, and (ii) the multi-channel case $(K>1)$, where we assume that the channels are orthogonal to each other. In the numerical evaluations, when $K>1$ we adopt $K=52$. We use $k$ to denote the channel index. When $K=1$, we omit the indices.

$P_{u, k}$ denotes the transmission power level at station $u \in$ $\{m, b\}$ on channel $k$ and $\overline{P_{u}}$ denotes the maximum sum of transmission power levels at station $u$ : $\sum_{k=1}^{K} P_{u, k} \leq \overline{P_{u}}$, where $u \in\{m, b\}$. For simplicity, we introduce notation for the normalized transmission power levels: $\alpha_{b, k}=P_{b, k} / \overline{P_{b}}$, $\alpha_{m, k}=P_{m, k} / \overline{P_{m}}$. The constraints for the sum of transmission power levels are then: $\sum_{k} \alpha_{b, k} \leq 1$ and $\sum_{k} \alpha_{m, k} \leq 1$.

$\overline{\gamma_{b m, k}}$ and $\overline{\gamma_{m b, k}}$ denote the SNR of the signal from the $\mathrm{BS}$ to the MS and from the MS to the BS, respectively, on channel $k$, when the transmission power level on channel $k$ is set to its maximum value $\left(\overline{P_{b}}, \overline{P_{m}}\right.$, respectively). $\overline{\gamma_{b m}} \equiv \frac{1}{K} \sum_{k} \overline{\gamma_{b m, k}} / K$ and $\overline{\gamma_{m b}} \equiv \frac{1}{K} \sum_{k} \overline{\gamma_{m b, k}} / K$ denote the average SNR when the power levels are equally allocated over channels (i.e., when $\alpha_{b, 1}=\ldots=\alpha_{b, K}=1 / K$ and $\left.\alpha_{m, 1}=\ldots=\alpha_{m, K}=1 / K\right)$. In the numerical evaluations, we adopt $\overline{\gamma_{b m, k}}=K \overline{\gamma_{b m}}$ and $\overline{\gamma_{m b, k}}=K \overline{\gamma_{m b}}, \forall k$, to focus on the effects caused by FD operation. Our results, however, hold for general values of $\overline{\gamma_{b m, k}}$ and $\overline{\gamma_{m b, k}}$ over channels $k$.

Similarly to $[7,16,17]$, we model the remaining SI on channel $k$ as a constant fraction of the transmission power level on channel $k$. The Self-Interference-to-Noise-Ratio (XINR) at the BS on channel $k$ when $\alpha_{b, k}=1$ is denoted by $\overline{\gamma_{b b, k}}$. The XINR at the MS on channel $k$ when $\alpha_{m, k}=1$ is denoted by $\overline{\gamma_{m m, k}}$. In the numerical evaluations of the multi-channel case, we use $\overline{\gamma_{b b, k}} / K=1=0 \mathrm{~dB}$, as shown in Fig. 2(a), which is motivated by [5]. For $\overline{\gamma_{m m, k}}$, we consider three FD RFIC designs from [24], shown in Fig. 2(b)-(d). For the FD RFICs from [24], we assume additional $50 \mathrm{~dB}$ of cancellation in the digital domain and $110 \mathrm{~dB}$ difference between the maximum transmission signal and the noise.

For the DL rate on channel $k, r_{b, k}$, and for the UL rate on channel $k, r_{m, k}$, we use the Shannon capacity formula: $r_{b, k}=\log \left(1+\frac{\alpha_{b, k} \overline{\gamma_{b m, k}}}{1+\alpha_{m, k} \overline{\gamma_{m m, k}}}\right), r_{m, k}=\log \left(1+\frac{\alpha_{m, k} \overline{\gamma_{m b, k}}}{1+\alpha_{b, k} \bar{\gamma}_{b b, k}}\right)$, where log denotes the base-2 logarithm. $r_{b}=\sum_{k} r_{b, k}$ denotes the sum of DL rates over channels $k, r_{m}=\sum_{k} r_{m, k}$ denotes the sum of UL rates over channels $k$, and $r=r_{m}+r_{b}$ denotes the sum of all UL and DL rates over channels $k$ (in the following, we refer to $r$ as the sum rate).

We denote by $\overline{r_{b}}=\max \left\{r_{b}\left(\left\{\alpha_{b, k}\right\},\left\{\alpha_{m, k}\right\}\right): \sum_{k} \alpha_{b, k} \leq 1\right.$, $\left.\sum_{k} \alpha_{m, k} \leq 1\right\}$ the maximum DL rate. Observe that when

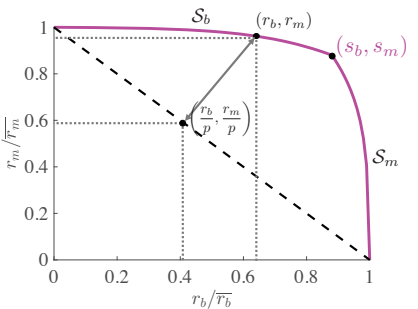

(a)

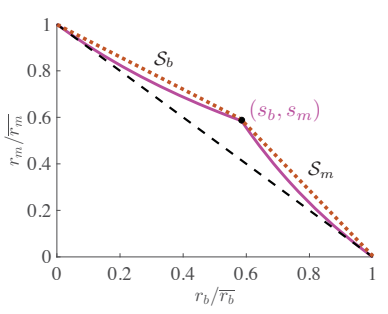

(b)
Figure 3: (a) Convex and (b) non-convex FD capacity regions. A dashed line delimits the corresponding TDD region. An FD region is convex, if and only if segments $\mathcal{S}_{b}$ (connecting $\left(0, \overline{r_{m}}\right)$ and $\left(s_{b}, s_{m}\right)$ ) and $\mathcal{S}_{m}$ (connecting $\left(s_{b}, s_{m}\right)$ and $\left.\left(\overline{r_{b}}, 0\right)\right)$ can be represented by a concave function $r_{m}\left(r_{b}\right)$.

$r_{b}$ is maximized, we have $\sum_{k} \alpha_{b, k}=1, \alpha_{m, k}=0, \forall k$, i.e., $\overline{r_{b}}$ is equal to the maximum HD rate on the DL. Similarly, $\overline{r_{m}}$ denotes the maximum UL rate.

A capacity region of an FD link is the set of all achievable UL-DL FD rate pairs. Examples of FD regions appear in Fig. 3, where a full line represents the FD region boundary, and a dashed line represents the TDD region boundary. The problem of determining the FD capacity region is the problem of maximizing one of the rates (e.g., $r_{m}$ ) when the other rate $\left(r_{b}\right)$ is fixed, subject to the sum power constraints.

An FD capacity region is not necessarily convex. In such cases, we also consider a convexified or TDFD capacity region, namely, the convex hull of the FD capacity region. In practice, the TDFD region would correspond to time sharing between different FD rate pairs. Fig. 3(b) illustrates a nonconvex FD capacity region, with the dotted line representing the boundary of the TDFD capacity region.

To compare an FD or a TDFD capacity region to its corresponding TDD region, we use the following definition (a similar definition appears in [17], see Fig. 3(a) for intuition):

Definition 3.1. For a given rate pair $\left(r_{b}, r_{m}\right)$ from an FD or TDFD capacity region, the rate improvement $p$ is defined as the largest (positive) number such that $\left(\frac{r_{b}}{p}, \frac{r_{m}}{p}\right)$ is at the boundary of the corresponding TDD capacity region.

Using simple geometry, $p$ can be computed as follows [17]:

Proposition 3.2. $p\left(r_{b}, r_{m}\right)=r_{b} / \overline{r_{b}}+r_{m} / \overline{r_{m}}$.

\section{SINGLE CHANNEL}

We now study the structural properties of the FD and TDFD capacity regions for a single FD channel and devise 
an algorithm that determines the points at the boundary of the TDFD capacity region. First, we provide structural results that characterize $\mathrm{FD}$ capacity regions. We prove that the FD region boundary, which can be described by a function $r_{m}\left(r_{b}\right)$, can only have up to four either convex or concave pieces that can only appear in certain specific arrangements. We also provide necessary and sufficient conditions for the region's boundary to take one of the possible shapes. As a corollary, we derive necessary and sufficient conditions for the FD region to be convex as a function of $\overline{\gamma_{b m}}, \overline{\gamma_{m b}}, \overline{\gamma_{m m}}$, and $\overline{\gamma_{b b}}$. Based on the structural results, we present a simple and fast algorithm that can determine any point at the boundary of the TDFD capacity region. For a given rate $r_{b}^{*}$, to find the maximum rate $r_{m}$ subject to $r_{b}=r_{b}^{*}$, the algorithm determines the shape of the capacity region as a function of $\overline{\gamma_{b m}}, \overline{\gamma_{m b}}, \overline{\gamma_{m m}}$, and $\overline{\gamma_{b b}}$, and either directly computes $r_{m}$ or performs a binary search to find it.

\subsection{Capacity Region Structural Results}

We state all the results in this section for the problem of finding $r_{m}\left(r_{b}\right)$ when $r_{b}=r_{b}^{*}$. The results for maximizing $r_{b}\left(r_{m}\right)$ when $r_{m}=r_{m}^{*}$ follow by symmetric arguments. We start by characterizing the power allocation at the boundary of an FD capacity region, given by the following simple proposition (used implicitly in [17]). The proof appears in [18]. In the rest of the section, $s_{b}=r_{b}(1,1)$, $s_{m}=r_{m}(1,1)$.

Proposition 4.1. If $r_{b}=r_{b}^{*} \leq s_{b}$, then $r_{m}$ is maximized for $\alpha_{m}=1$ and $\alpha_{b}$ that solves $r_{b}\left(\alpha_{b}, 1\right)=r_{b}^{*}$.

Proposition 4.1 implies that to determine any point $\left(r_{b}, r_{m}\right)$ at the boundary of the capacity region, where $r_{b}, r_{m}>0$, for $r_{b} \leq s_{b}$ (resp. $r_{m} \leq s_{m}$ ), it suffices to find $\alpha_{b}$ (resp. $\alpha_{m}$ ) that satisfies $r_{b}=r_{b}\left(\alpha_{b}, 1\right)$ (resp. $r_{m}=r_{m}\left(1, \alpha_{m}\right)$ ). The capacity region is convex, if and only if (i) $r_{b}\left(r_{m}\right)$ is concave for $r_{m} \in\left(0, s_{m}\right]$ and $r_{b}$ at the boundary of the capacity region, (ii) $r_{m}\left(r_{b}\right)$ is concave for $r_{b} \in\left(0, s_{b}\right]$ and $r_{m}$ at the boundary of the capacity region, and (iii) the functions $r_{m}\left(r_{b}\right)$ and $r_{b}\left(r_{m}\right)$ intersect at $\left(s_{b}, s_{m}\right)$ under an angle smaller than $\pi$.

If the FD capacity region is convex (Fig. 3(a)), then to maximize $r_{m}$ subject to $r_{b}=r_{b}^{*}$, it is always optimal to use FD and allocate the power levels according to Proposition 4.1. This is not necessarily true, if the capacity region is not convex; in that case, it may be optimal to use a time-sharing scheme between two FD rate pairs (TDFD), since a convex combination of e.g., $\left(s_{b}, s_{m}\right)$ and $\left(\overline{r_{b}}, 0\right)$ may lie above the FD capacity region boundary (e.g., Fig. 3(b)).

The following lemma characterizes the FD capacity region boundary (the proof appears in [18]).

Lemma 4.2. Given positive $\overline{\gamma_{m b}}, \overline{\gamma_{b m}}, \overline{\gamma_{b b}}, \overline{\gamma_{m m}}$, let $r_{m}\left(r_{b}\right)$ describe the boundary of the FD capacity region for $r_{b} \in$ $\left[0, s_{b}\right]$, and $r_{b}\left(r_{m}\right)$ describe the boundary of the FD capacity region for $r_{m} \in\left[0, s_{m}\right]$. Then $r_{m}\left(r_{b}\right)\left(r_{b} \in\left[0, s_{b}\right]\right)$ and $r_{b}\left(r_{m}\right) \quad\left(r_{m} \in\left[0, s_{m}\right]\right)$ can only be described by one of the following three function types: (i) concave, (ii) convex, and (iii) concave for $r_{b} \in\left[0, r_{b}^{+}\right]$for some $r_{b}^{+}<s_{b}$ in the case of $r_{m}\left(r_{b}\right)$, concave for $r_{m} \in\left[0, r_{m}^{+}\right]$for some $r_{m}^{+}<s_{m}$ in the case of $r_{b}\left(r_{m}\right)$, and convex on the rest of the domain.

The following corollary of the proof of Lemma 4.2 gives necessary and sufficient conditions for $r_{m}\left(r_{b}\right)$ to be concave for $r_{b} \in\left[0, s_{b}\right]$ (the proof appears in [18]).

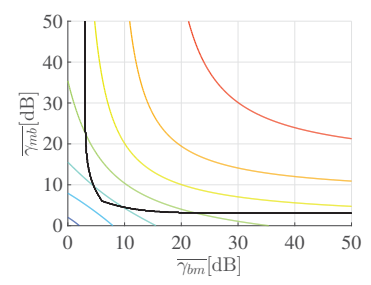

(a) $\overline{\gamma_{m m}}=0 \mathrm{~dB}$

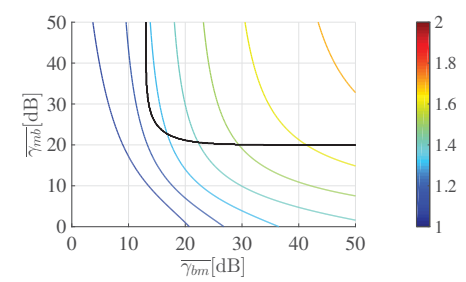

(b) $\overline{\gamma_{m m}}=10 \mathrm{~dB}$
Figure 4: Convexity of the capacity region vs. rate improvement for $\overline{\gamma_{b b}}=0 \mathrm{~dB}$ and: (a) $\overline{\gamma_{m m}}=0 \mathrm{~dB}$ and (b) $\overline{\gamma_{m m}}=10 \mathrm{~dB}$. The capacity region is convex for UL and DL SNRs north and east from the black curve.

Corollary 4.3. For given positive $\overline{\gamma_{m b}}, \overline{\gamma_{b m}}, \overline{\gamma_{b b}}$, and $\overline{\gamma_{m m}}$, $r_{m}\left(r_{b}\right)$ is concave for $r_{b} \in\left[0, s_{b}\right]$ if and only if:

$$
\begin{gathered}
\overline{\gamma_{b m}}>\max \left\{\left(\overline{\gamma_{m m}}\right)^{2}-1, \overline{\gamma_{b b}}\left(1+\overline{\gamma_{m m}}\right) \frac{2+\overline{\gamma_{m b}}}{1+\overline{\gamma_{m b}}},\right. \\
\left.\left(1+\overline{\gamma_{m m}}\right) \frac{2+\left(2+\overline{\gamma_{m b}}\right) / \overline{\gamma_{b b}}}{\left(1+\overline{\gamma_{m b}}\right) /\left(\overline{\gamma_{b b}}\right)^{2}-1}\right\} .
\end{gathered}
$$

Finally, we show that whenever both $r_{m}\left(r_{b}\right)$ is concave for all $r_{b} \in\left[0, s_{b}\right]$ and $r_{b}\left(r_{m}\right)$ is concave for all $r_{m} \in\left[0, s_{m}\right]$, the FD region is convex (the proof of the proposition is in [18]).

Proposition 4.4. If both $r_{m}\left(r_{b}\right)$ is concave for all $r_{b} \in$ $\left[0, s_{b}\right]$ and $r_{b}\left(r_{m}\right)$ is concave for all $r_{m} \in\left[0, s_{m}\right]$, then the FD capacity region is convex.

Fig. 4 illustrates the regions of (maximum) SNR values $\overline{\gamma_{b m}}$ and $\overline{\gamma_{m b}}$ for which the FD capacity region is convex, for different values of $\overline{\gamma_{m m}}$ and $\overline{\gamma_{b b}}$, compared to the maximum achievable rate improvements. The black line delimits the region of $\overline{\gamma_{b m}}$ and $\overline{\gamma_{m b}}$ for which the $\mathrm{FD}$ region is convex: north and east from it, the region is convex, while south and west from it, the region is not convex. As Fig. 4 suggests, high $($ over $1.6 \times$ ) rate improvements are mainly achievable in the area where the FD region is convex, unless one of the SNR values $\overline{\gamma_{b m}}$ and $\overline{\gamma_{m b}}$ is much higher than the other.

\subsection{Determining TDFD Capacity Region}

We now turn to the problem of allocating UL and DL rates, possibly through a combination of FD and TDD, which is equivalent to determining the TDFD capacity region. As before, the problem is to maximize $r_{m}$ subject to $r_{b}=r_{b}^{*}$ and the power constraints. Denote the maximum $r_{m}$ such that $r_{b}=r_{b}^{*}$ as $r_{m}^{*}$. From Lemma 4.2 , there can be 3 cases: Case 1: $r_{m}\left(r_{b}\right)$ is concave for all $r_{b} \in\left[0, s_{b}\right]$. From concavity of $r_{m}\left(r_{b}\right), r_{m}^{*}=r_{m}\left(\alpha_{b}, 1\right)$, where $\alpha_{b}$ solves $r_{b}\left(\alpha_{b}, 1\right)=r_{b}^{*}$. Case 2: $r_{m}\left(r_{b}\right)$ is convex for all $r_{b} \in\left[0, s_{b}\right]$. Using convexity, if the rate improvement at $\left(s_{b}, s_{m}\right)$ is less than 1 and $r_{b}\left(r_{m}\right)$, $r_{m} \in\left[0, s_{m}\right]$, is convex, it is optimal to use TDD. If the rate improvement is at least 1 , it is optimal to place $r_{m}^{*}$ on the line connecting $\left(0, \overline{r_{m}}\right)$ and $\left(s_{b}, s_{m}\right)$. If $r_{b}\left(r_{m}\right)$ for $r_{m} \in\left[0, s_{m}\right]$ is concave for $r_{m} \in\left[0, r_{m}^{+}\right]$, where $r_{m}^{+} \leq s_{b}$, and the rate improvement at $\left(s_{b}, s_{m}\right)$ is less than $1, r_{m}^{*}$ will lie on the boundary of the TDFD, but not FD, capacity region.

Case 3: $r_{m}\left(r_{b}\right)$ is strictly concave for $r_{b} \in\left[0, r_{b}^{+}\right)$, strictly convex for $r_{b} \in\left(r_{b}^{+}, s_{b}\right]$, and $\frac{d r_{m}}{d r_{b}}=0$ at $r_{b}=r_{b}^{+}$, where $r_{b}^{+}<s_{b}$. Then, $r_{m}^{*}$ may lie either on (the boundary of) FD or TDFD capacity region, even if $r_{b}^{*} \leq r_{b}^{+}$. 


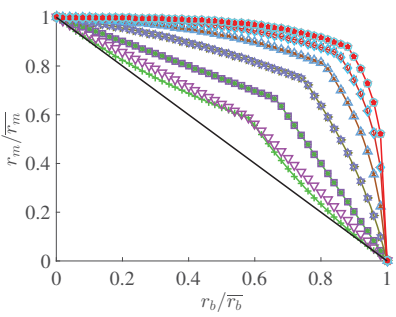

(a) $\overline{\gamma_{b b}}=0 \mathrm{~dB}, \overline{\gamma_{m m}}=0 \mathrm{~dB}$

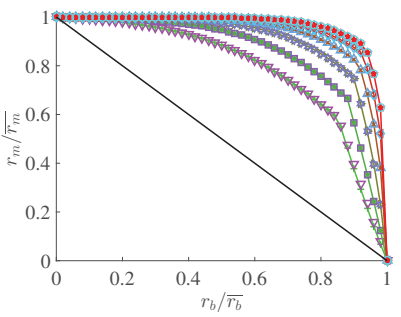

(d) $\overline{\gamma_{b b}}=0 \mathrm{~dB}, \overline{\gamma_{m m}}=0 \mathrm{~dB}$

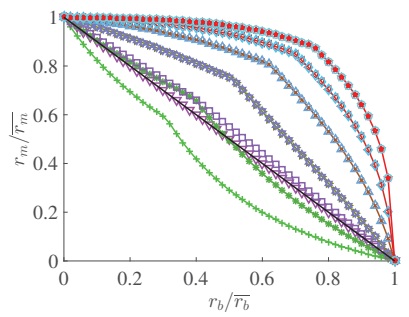

(b) $\overline{\gamma_{b b}}=0 \mathrm{~dB}, \overline{\gamma_{m m}}=5 \mathrm{~dB}$

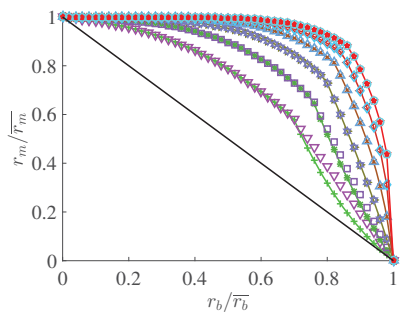

(e) $\overline{\gamma_{b b}}=0 \mathrm{~dB}, \overline{\gamma_{m m}}=5 \mathrm{~dB}$

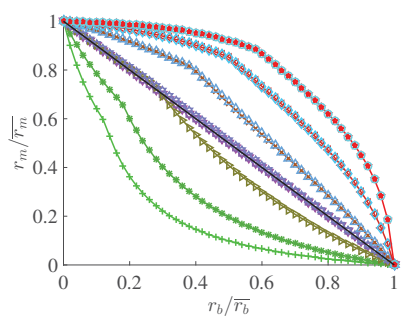

(c) $\overline{\gamma_{b b}}=0 \mathrm{~dB}, \overline{\gamma_{m m}}=10 \mathrm{~dB}$

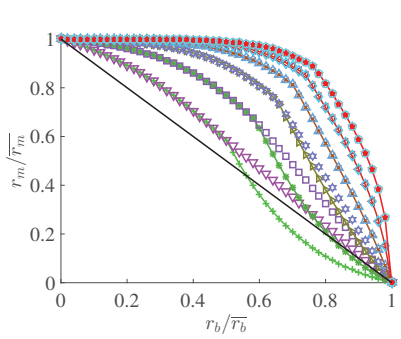

(f) $\overline{\gamma_{b b}}=0 \mathrm{~dB}, \overline{\gamma_{m m}}=10 \mathrm{~dB}$ $+\overline{\gamma_{m b}}=\overline{\gamma_{b m}}=0 \mathrm{~dB}, \mathrm{FD}$

$\nabla \overline{\gamma_{m b}}=\overline{\gamma_{b m}}=0 \mathrm{~dB}, \mathrm{FD}+\mathrm{TDD}$ $-\overline{\gamma_{m b}}=\overline{\gamma_{b m}}=5 \mathrm{~dB}, \mathrm{FD}$

$\square \overline{\gamma_{m b}}=\overline{\gamma_{b m}}=5 \mathrm{~dB}, \mathrm{FD}+\mathrm{TDD}$ $\rightarrow \overline{\gamma_{m b}}=\overline{\gamma_{b m}}=10 \mathrm{~dB}, \mathrm{FD}$

$\not \overline{\gamma_{m b}}=\overline{\gamma_{b m}}=10 \mathrm{~dB}, \mathrm{FD}+\mathrm{TDD}$

$\# \frac{\gamma m b}{\gamma m b}=\frac{\gamma m}{\gamma m}=15 \mathrm{~dB}, \mathrm{FD}$

$* \frac{m b}{\gamma m b}=\overline{\gamma m}=15 \mathrm{BB}, \mathrm{FD}+\mathrm{m}$

$\Delta \overline{\gamma_{m b}}=\overline{\gamma_{b m}}=15 \mathrm{~dB}, \mathrm{FD}+\mathrm{TDD}$

$\varangle \overline{\gamma_{m b}}=\overline{\gamma_{b m}}=20 \mathrm{~dB}, \mathrm{FD}$

$\diamond \overline{\gamma_{m b}}=\overline{\gamma_{b m}}=20 \mathrm{~dB}, \mathrm{FD}+\mathrm{TDD}$

$\star \overline{\gamma_{m b}}=\overline{\gamma_{b m}}=25 \mathrm{~dB}, \mathrm{FD}$

$\overline{\gamma_{m b}}=\overline{\gamma_{b m}}=25 \mathrm{~dB}, \mathrm{FD}+\mathrm{TDD}$ -TDD

Figure 5: Capacity regions for (a)-(c) $\overline{\gamma_{b m}}=\overline{\gamma_{m b}}$ and (d)-(f) $\overline{\gamma_{b m}}>\overline{\gamma_{m b}}$.

To determine the optimal $r_{m}^{*}$ in Cases 2 and 3, we need to "convexify" the capacity region. Fortunately, the problem has enough structure so that this "convexification" can be done efficiently. We show the following propositions, which will lead to the convexified region. The proofs appear in [18].

Proposition 4.5. If $\left(s_{b}, s_{m}\right)$ maximizes the sum of $U L$ and $D L$ rates, then $\left(s_{b}, s_{m}\right) \geq \lambda\left(r_{b}^{\prime}, r_{m}^{\prime}\right)+(1-\lambda)\left(r_{b}^{\prime \prime}, r_{m}^{\prime \prime}\right)$ element-wise for any $\lambda \in[0,1]$, and any two feasible rate pairs $\left(r_{b}^{\prime}, r_{m}^{\prime}\right)$ and $\left(r_{b}^{\prime \prime}, r_{m}^{\prime \prime}\right)$.

Proposition 4.6. If $s_{b}+s_{m}<\overline{r_{m}}$, then $r_{m}\left(r_{b}\right)$ is convex on the entire segment from $\left(0, \overline{r_{m}}\right)$ to $\left(s_{b}, s_{m}\right)$.

Now we are ready to handle Case 3 and the last part of Case 2, in the following lemma. The proof of the lemma is constructive, i.e., it describes the algorithm for determining the TDFD capacity region, and can be found in [18]. The algorithm uses Lemma 4.2 to determine the shape of the capacity region, and then, relying on Propositions 4.5 and 4.6, performs at most two binary searches. Note that the convexification needs to be performed only once; after that, $r_{m}\left(r_{b}\right)$ (and $r_{b}\left(r_{m}\right)$ ) can be represented in a black-box manner, requiring constant computation to determine any rate pair $\left(r_{b}^{*}, r_{m}^{*}\right)$, given either $r_{b}^{*}$ or $r_{m}^{*}$.

LEMMA 4.7. The boundary of the TDFD capacity region can be determined in time $O\left(\log \left(\varepsilon^{-1} \overline{r_{b}}\right)\right)$, where $\varepsilon$ is the additive error of $r_{m}^{*}=\max \left\{r_{m}: r_{b}=r_{b}^{*}\right\}$, and the binary search, if employed, takes at most $\left\lceil\log \left(\varepsilon^{-1} \cdot 1.4 \overline{r_{b}}\right)\right\rceil$ steps.

Using the methods mentioned above, FD and TDFD capacity regions were obtained for different combinations of $\overline{\gamma_{b m}}, \overline{\gamma_{m b}}, \overline{\gamma_{m m}}$, and $\overline{\gamma_{b b}}$ (Fig. 5). As expected, as $\overline{\gamma_{m m}}$ increases and $\overline{\gamma_{m b}}$ and $\overline{\gamma_{b m}}$ decrease, the rate improvements decrease and more FD regions become non-convex.

\section{MULTI-CHANNEL - FIXED POWER}

In this section, we consider the problem of determining FD and TDFD capacity regions over multiple channels when the (shape of) the power allocation is fixed, but the total transmission power level can be varied. We first provide characterization of the FD capacity region, which allows computing any point on the FD capacity region via a binary search. Then, we turn to the problem of determining the TDFD capacity region. Due to the lack of structure as in the single channel case, in the multi-channel case the TDFD capacity region cannot in general be determined by a binary search. We argue, however, that for inputs that are relevant in practice this problem can be solved in real time.

\subsection{Capacity Region}

Suppose that we want to determine the FD capacity region, given a fixed power allocation over $K$ orthogonal channels: $\alpha_{b, 1}=\alpha_{b, 2}=\ldots=\alpha_{b, K} \equiv \alpha_{b}$ and $\alpha_{m, 1}=\alpha_{m, 2}=$ $\ldots=\alpha_{m, K} \equiv \alpha_{m}$. Note that setting the power allocation so that all $\alpha_{b, k}$ 's and all $\alpha_{m, k}$ 's are equal is without loss of generality, since we can represent an arbitrary fixed power allocation in this manner by appropriately scaling the values of $\overline{\gamma_{b m}}, \overline{\gamma_{m b}}, \overline{\gamma_{m m}}$, and $\overline{\gamma_{b b}}$. The sum of the UL and DL rates over the (orthogonal) channels can then be written as $r=r_{b}+r_{m}$, where: $r_{b}=\sum_{k=1}^{K} \log \left(1+\frac{\alpha_{b} \overline{\gamma_{b m, k}}}{1+\alpha_{m} \overline{\gamma_{m m, k}}}\right)$ and $r_{m}=\sum_{k=1}^{K} \log \left(1+\frac{\alpha_{m} \overline{\gamma_{m b, k}}}{1+\alpha_{b} \overline{\gamma_{b b}, k}}\right)$. Let $s_{b}=r_{b}\left(\alpha_{b}=\frac{1}{K}, \alpha_{m}=\right.$ $\left.\frac{1}{K}\right), s_{m}=r_{m}\left(\alpha_{b}=\frac{1}{K}, \alpha_{m}=\frac{1}{K}\right)$. We characterize the FD capacity region in the following lemma (the proof is in [18]).

Lemma 5.1. For a fixed $r_{b}=r_{b}^{*} \leq s_{b}, r_{m}$ is maximized for $\alpha_{m}=1 / K$.

Using Lemma 5.1, we can construct the entire FD capacity region by solving (i) $r_{b}=r_{b}^{*}$ for $\alpha_{b}$, when $\alpha_{m}=1 / K$ and $r_{b}^{*} \in\left[0, s_{b}\right]$, and (ii) $r_{b}=r_{b}^{*}$ for $\alpha_{m}$, when $\alpha_{b}=1 / K$ and $r_{b}^{*} \in\left(s_{b}, \bar{r}_{b}\right]$. Note that $r_{b}=r_{b}^{*}$ can be solved for $\alpha_{b}$ when $r_{b} \in\left[0, s_{b}\right]$ (resp. for $\alpha_{m}$ ) by using a binary search, since $r_{b}$ is monotonic and bounded in $\alpha_{b}$ for $r_{b} \in\left[0, s_{b}\right]$ (resp. $\alpha_{m}$ for $\left.r_{b} \in\left(s_{b}, \overline{r_{b}}\right]\right)$. The pseudocode is provided in Algorithm 1 (MCFInD $-r_{m}$ ). The bound on the running time is provided in Proposition 5.2, whose proof is in [18]. 


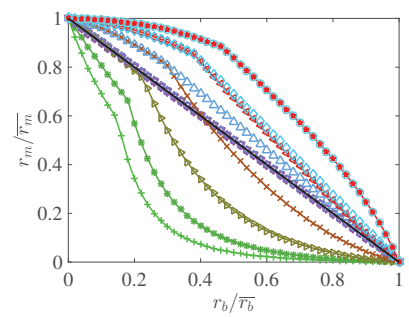

(a)

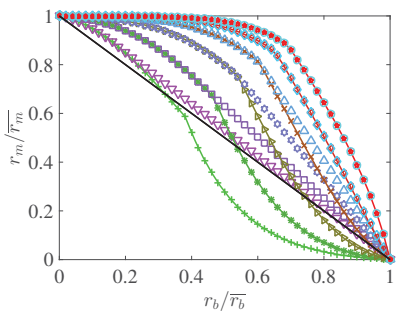

(d)

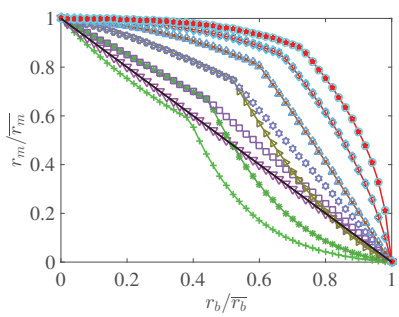

(b)

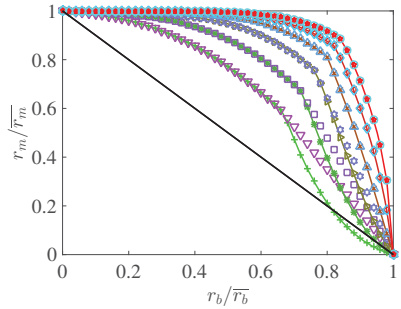

(e)
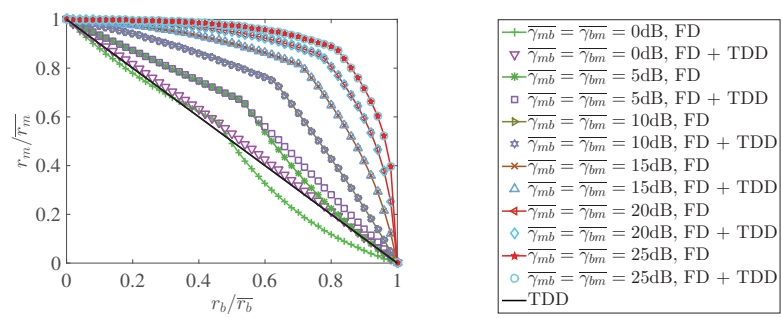

(c)

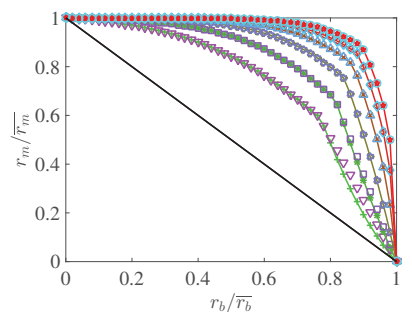

(f)

Figure 6: Capacity regions for $\overline{\gamma_{b b, k}}$ from Fig. 2(a), and $\overline{\gamma_{m m, k}}$ from (a), (d) Fig. 2(b), (b), (e) Fig. 2(c), and (c), (f) Fig. 2(d).

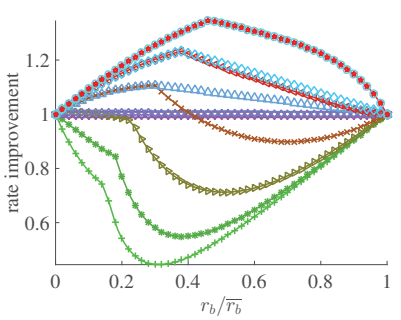

(a)

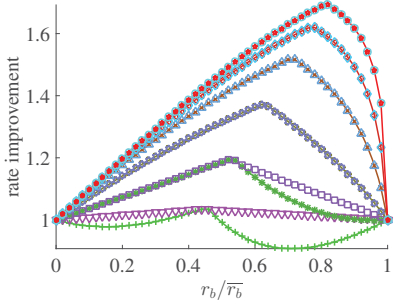

(b)

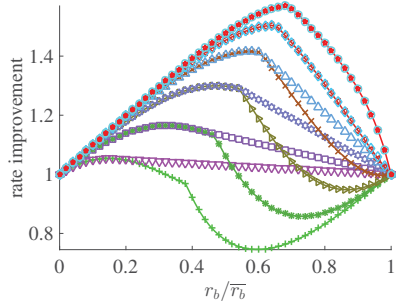

(c)

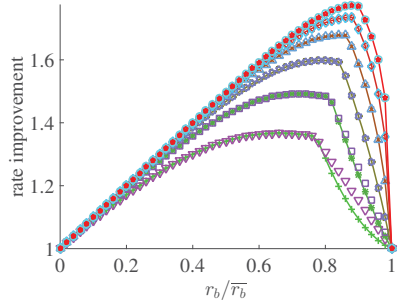

(d)

Figure 7: Rate improvements corresponding to capacity regions from (a) Fig. 6(a), (b) Fig. 6(c),(c) Fig. 6(d), and (d) Fig. 6(f).

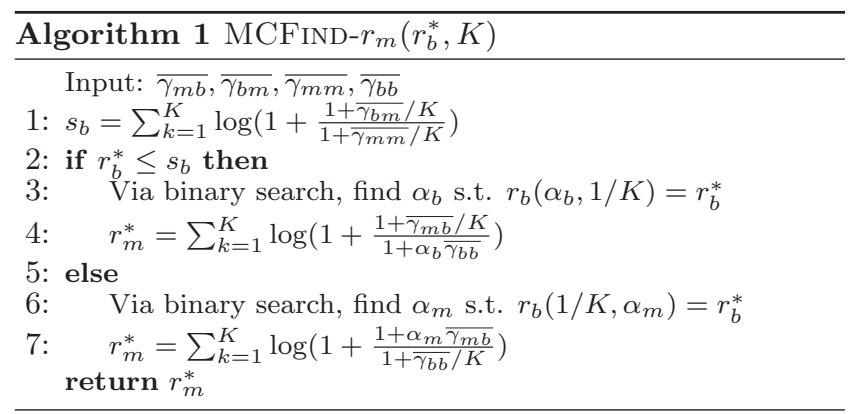

Proposition 5.2. The running time of MCFInd- $r_{m}$ is $O\left(K \log \left(\sum_{k} \frac{\overline{\gamma_{b b, k}}}{K \varepsilon}\right)\right)$, where $\varepsilon$ is the additive error for $r_{m}^{*}$.

Notice that in practice $\overline{\gamma_{b b, k}} / K \leq 1, \overline{\gamma_{m m, k}} / K \leq 100$, and $K$ is at the order of 100 , which makes the running time of MCFIND- $r_{m}$ suitable for a real-time implementation.

Unlike in the single channel case, where the shape of the FD region boundary is very structured, in the multi-channel case the region does not necessarily have the property that $r_{m}\left(r_{b}\right)$ (and $r_{b}\left(r_{m}\right)$ ) has at most one concave and at most one convex piece (for a more through discussion, see [18]).

Although in general the problem of convexifying the FD region seems difficult in the multi-channel case, in practice it can be solved efficiently. The reason is that in $\mathrm{Wi}-\mathrm{Fi}$ and cellular networks the output power levels take values from a discrete set of size $N$, where $N<100$. Therefore, (for fixed $\left.\overline{\gamma_{m b, k}}, \overline{\gamma_{b m, k}}, \overline{\gamma_{b b, k}}, \overline{\gamma_{m m, k}}, \forall k\right) r_{b}$ can take at most $N$ distinct values. To find the TDFD capacity region, since the points of the FD region are determined in order increasing in $r_{b}, \Theta(N)$ computation suffices (Ch. 33, [9]).

The capacity regions and the rate improvements for $\overline{\gamma_{b b, k}}$ described by Fig. 2(a) and the three cases of $\overline{\gamma_{m m, k}}$ described by Fig. 2(b)-(d), for equal power allocation and equal SNR over channels, are shown in Fig. 6 and 7, respectively. As the cancellation becomes more broadband, namely as $\overline{\gamma_{m m, k}}$ 's change from those described in Fig. 2(b) over 2(c) to 2(d), the rate improvements become higher and the capacity region becomes convex for lower values of $\overline{\gamma_{m b}}$ and $\overline{\gamma_{b m}}$.

\section{MULTI-CHANNEL - GENERAL POWER}

We now consider the computation of TDFD capacity regions under general power allocations. In this case there are $2 K$ variables $\left(\alpha_{b, 1}, \ldots, \alpha_{b, K}, \alpha_{m, 1}, \ldots, \alpha_{m, K}\right)$, compared to 2 variables $\left(\alpha_{b}\right.$ and $\left.\alpha_{m}\right)$ from the previous section.

Computing $r_{m}^{*}=\max \left\{r_{m}: r_{b}=r_{b}^{*}\right\}$ is a non-convex problem, and is hard to optimize in general. Yet, we present an algorithm that is guaranteed to converge to a stationary point, under certain restrictions. In practice, the stationary point to which it converges is also a global maximum. The restrictions are based on [17] and they guarantee that $r_{b}+$ 
$r_{m}$ is concave when either the $\alpha_{b, k}$ 's or $\alpha_{m, k}$ 's are fixed. Note that the restrictions do not make the problem $r_{m}^{*}=$ $\max \left\{r_{m}: r_{b}=r_{b}^{*}\right\}$ convex (see Section 6.1). The restrictions are mild in the sense that they do not affect the optimum by much whenever $\overline{\gamma_{b m, k}}$ and $\overline{\gamma_{m b, k}}$ do not differ much.

Though for many practical cases the algorithm is nearoptimal and runs in polynomial time, its running time in general is not suitable for a real-time implementation. To combat the high running time, in Section 6.2 we develop a simple heuristic that in most cases has similar performance.

\subsection{Capacity Region}

Determining the FD region under a general power allocation is equivalent to solving $\left\{\max r_{m}: r_{b}=r_{b}^{*}\right\}$ for any $r_{b}^{*} \in\left[0, \overline{r_{b}}\right]$ over $\alpha_{b, k}, \alpha_{m, k} \geq 0, \sum_{k} \alpha_{b, k} \leq 1, \sum_{k} \alpha_{m, k} \leq 1$. It is not hard to show that $\frac{d r_{m}}{d r_{b}}<0$, and, therefore, the problem is equivalent to $(P)=\left\{\max r_{m}: r_{b} \geq r_{b}^{*}\right\}$.

Problem $(P)$ is not convex, even when some of the variables are fixed. When the $\alpha_{m, k}$ 's are fixed, $r_{b}$ is concave in $\alpha_{b, k}$ 's and the feasible region is convex, however, $r_{m}$ is convex as well. Conversely, when the $\alpha_{b, k}$ 's are fixed, $r_{m}$ is concave in $\alpha_{m, k}$ 's, but the feasible region is not convex since $r_{b}$ is convex in $\alpha_{m, k}$ 's. Therefore, the natural approach to determining the FD region fails.

On the other hand, [17] provides conditions that guarantee that $\forall k, r=r_{b}+r_{m}$ is (i) concave and increasing in $\alpha_{m, k}$ when $\alpha_{b, k}$ is fixed, and (ii) concave and increasing in $\alpha_{b, k}$ when $\alpha_{m, k}$ is fixed. These conditions are not very restrictive: when they cannot be satisfied, one cannot gain much from FD additively - the additive gain is less than $1 \mathrm{~b} / \mathrm{s} / \mathrm{Hz}$ compared to the maximum of the UL and DL rates. However, these conditions can be very restrictive when the difference between $\overline{r_{b}}$ and $\overline{r_{m}}$ is high. The conditions are:

$$
\begin{aligned}
& \overline{\gamma_{b m, k}} \geq \overline{\gamma_{b b, k}}\left(1+\alpha_{m, k} \overline{\gamma_{m m, k}}\right), \forall k \\
& \overline{\gamma_{m b, k}} \geq \overline{\gamma_{m m, k}}\left(1+\alpha_{b, k} \overline{\gamma_{b b, k}}\right), \forall k .
\end{aligned}
$$

Notice that when $\overline{\gamma_{b m, k}} \geq \overline{\gamma_{b b, k}}\left(1+\overline{\gamma_{m m, k}}\right)$ and $\overline{\gamma_{m b, k}} \geq$ $\overline{\gamma_{m m, k}}\left(1+\overline{\gamma_{b b, k}}\right)$, conditions (C1) and (C2) are non-restrictive (as they hold for any $\alpha_{b, k} \leq 1, \alpha_{m k} \leq 1$ ). When $\overline{\gamma_{b m, k}}<$ $\overline{\gamma_{b b, k}}$, (C1) cannot be satisfied for any $\alpha_{m, k}$ as $\alpha_{m, k} \geq 0$. Similarly for $\overline{\gamma_{m b, k}}<\overline{\gamma_{m m, k}}$, (C2) cannot hold for any $\alpha_{b, k}$.

We will use conditions $(\mathrm{C} 1)$ and $(\mathrm{C} 2)$ to formulate a new problem that is still non-convex, but more tractable than the original problem $(P)$. This way, we will get an upper bound on the capacity region and rate improvements when the conditions are non-restrictive. The new problem will also allow us to make a good estimate of the capacity region in the cases when $\overline{\gamma_{b m, k}}$ and $\overline{\gamma_{m b, k}}$ do not differ much.

Let $\left(s_{b}, s_{m}\right)$ denote the UL-DL rate pair that maximizes the sum of the rates over UL and DL channels.

LEMMA 6.1. If conditions (C1) and (C2) are non-restrictive, then, given $\overline{\gamma_{b m, k}}, \overline{\gamma_{m b, k}}, \overline{\gamma_{m m, k}}, \overline{\gamma_{b b, k}}$ for $k \in\{1, \ldots, K\}$, the TDFD capacity region can be determined by solving:

$$
(Q)=\left\{\begin{array}{ll}
\max & \sum_{k=1}^{K}\left(r_{b, k}\left(\alpha_{b, k}, \alpha_{m, k}\right)+r_{m, k}\left(\alpha_{b, k}, \alpha_{m, k}\right)\right) \\
\text { s.t. } & \sum_{k=1}^{K} r_{b, k}\left(\alpha_{b, k}, \alpha_{m, k}\right) \text { op } r_{b}^{*} \\
& \sum_{k=1}^{K} \alpha_{b, k} \leq 1, \sum_{k=1}^{K} \alpha_{m, k} \leq 1 \\
& \alpha_{b, k} \geq 0, \alpha_{m, k} \geq 0, \forall k
\end{array},\right.
$$

where op $=^{\prime} \leq^{\prime}$, if $r_{b}^{*} \leq s_{b}$ and op $=^{\prime} \geq '$, if $r_{b}^{*} \geq s_{b}$.

The proof of Lemma 6.1 is provided in [18].
When conditions (C1) and (C2) are restrictive, they provide upper bounds on $\alpha_{b, k}$ and $\alpha_{m, k}$ and they do not affect the optimal solution to $(Q)$ unless $\overline{\gamma_{b m, k}}>>\overline{\gamma_{m b, k}}$ or $\overline{\gamma_{m b, k}}>>\overline{\gamma_{b m, k}}$ for some $k$. To avoid infeasibility when restricting the feasible region of $(Q)$ by requiring $(\mathrm{C} 1)$ and (C2), similar to [17], we will set either $\alpha_{b, k}=0$ or $\alpha_{m, k}=0 .^{3}$

We write the restrictions imposed by $(\mathrm{C} 1)$ and $(\mathrm{C} 2)$ on the feasible region of $(Q)$ as follows, where $\alpha_{b, k} \leq A_{b}(k)$ and $\alpha_{m, k} \leq A_{m}(k), \forall k$. Notice that the restrictions are fixed for fixed $\overline{\gamma_{b m, k}}, \overline{\gamma_{m b, k}}, \overline{\gamma_{m m, k}}, \overline{\gamma_{b b, k}}$, and $r_{b}^{*}$. We refer to the restricted version of problem $(Q)$ as $\left(Q_{R}\right)$.

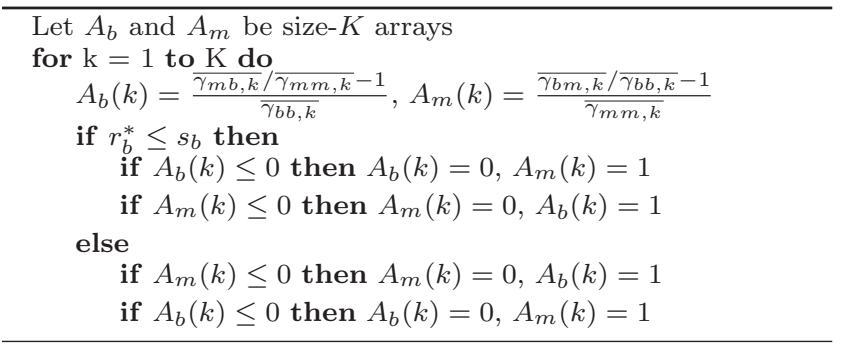

To solve $\left(Q_{R}\right)$, we will use a well-known practical method called alternating minimization (or maximization, as in our case) [19]. For a given problem $\left(P_{i}\right)$, the method partitions the variable set $x$ into two sets $x_{1}$ and $x_{2}$, and then iteratively applies the following procedure: (i) optimize $\left(P_{i}\right)$ over $x_{1}$ by treating the variables from $x_{2}$ as constants, (ii) optimize $\left(P_{i}\right)$ over $x_{2}$ by treating the variables from $x_{1}$ as constants, until a stopping criterion is reached.

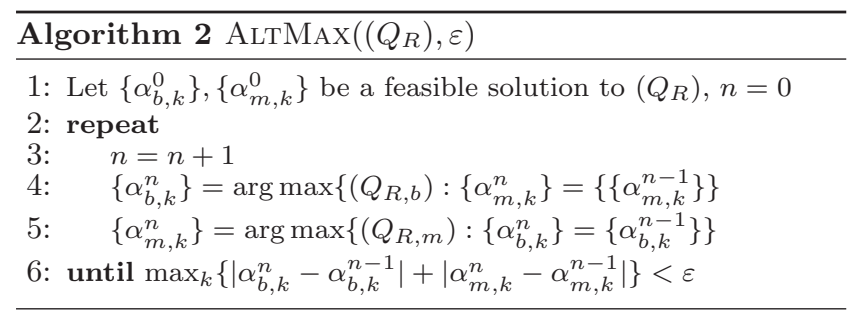

Even in the cases when $\left(P_{i}\right)$ is non-convex, if subproblems from (i) and (ii) have unique solutions and are solved optimally in each iteration, the method converges to a stationary point with rate $O(1 / \sqrt{n})$, where $n$ is the iteration count [4]. In the cases when, in addition, for each of the subproblems the objective is convex (concave for maximization problems), for each stationary point there exists an initial point such that the alternating minimization converges to that stationary point [11]. A common approach that works well in practice is to generate many random initial points and choose the best solution found. In our experiments, choosing $\alpha_{b, k}=\alpha_{m, k}=0$ as the initial point typically led to the best solution.

Due to the added restrictions in problem $\left(Q_{R}\right)$ imposed by $(\mathrm{C} 1)$ and $(\mathrm{C} 2)$, the objective in $\left(Q_{R}\right)$ is concave whenever either all $\alpha_{b, k}$ 's or all $\alpha_{m, k}$ 's are fixed, while the remaining variables are varied. Hence, our two subproblems for $Q_{R}$ will be: (i) $\left(Q_{R, b}\right)$, which is equivalent to $\left(Q_{R}\right)$ except that it treats $\alpha_{b, k}$ 's as variables and $\alpha_{m, k}$ 's as constants, and (ii) $\left(Q_{R, m}\right)$, which is equivalent to $\left(Q_{R}\right)$ except that it treats

\footnotetext{
${ }^{3}$ Recall that when $\alpha_{b, k}=0$, the sum of the rates is concave in $\alpha_{m, k}$ for any $\alpha_{m, k} \in[0,1]$. Similarly when $\alpha_{b, k}=0$.
} 
$\alpha_{m, k}$ 's as variables and $\alpha_{b, k}$ 's as constants. Given accuracy $\varepsilon$, the pseudocode is provided in Algorithm 2 (AltMax). The rate pair $\left(s_{b}, s_{m}\right)$ can be determined using the same algorithm by omitting the constraint $r_{b} \leq r_{b}^{*}$ (or $\left.r_{b} \geq r_{b}^{*}\right)$.

What remains to show is that both $\left(Q_{R, b}\right)$ and $\left(Q_{R, m}\right)$ have unique solutions that can be found in polynomial time. We do that in the following (constructive) lemma. Note that without the constraint $r_{b}^{*} \leq s_{b}$ or $r_{b}^{*} \geq s_{b}$, both $\left(Q_{R, b}\right)$ and $\left(Q_{R, m}\right)$ are convex and have strictly concave objectives, and therefore, we can determine $s_{b}$ using Altmax.

Lemma 6.2. Starting with a feasible solution $\left\{\alpha_{b, k}^{0}, \alpha_{m, k}^{0}\right\}$ to $\left(Q_{R}\right)$, in each iteration of ALTMAX the solutions to $\left(Q_{R, b}\right)$ and $\left(Q_{R, m}\right)$ are unique and can be found in polynomial time.

Proof. Suppose that $r_{b}^{*} \leq s_{b}$. Then it is not hard to verify that $\left(Q_{R, m}\right)$ is a convex problem with a strictly concave objective. The objective is strictly concave due to the enforcement of conditions (C1) and (C2), while all the constraints except for $r_{b} \leq r_{b}^{*}$ are linear. The constraint $r_{b} \leq r_{b}^{*}$ is convex as $r_{b}$ is convex in $\alpha_{m, k}$ 's. Therefore, $\left(Q_{R, m}\right)$ admits a unique solution that can be found in polynomial time through convex programming. By similar arguments, when $r_{b}^{*}>s_{b},\left(Q_{R, b}\right)$ admits a unique solution that can be found in polynomial time through convex programming.

Consider $\left(Q_{R, b}\right)$ when $r_{b}^{*} \leq s_{b}$. This problem is not convex due to the constraint $r_{b} \leq r_{b}^{*}$, as $r_{b}$ is concave in $\alpha_{b, k}$ 's. However, we will show that the problem has enough structure so that it is solvable in polynomial time.

Let $k^{*}=\arg \max _{k}\left\{\frac{\gamma_{b m, k}}{1+\alpha_{m, k} \bar{\gamma}_{m m, k}}-\gamma_{b b, k}+\frac{\gamma_{b b, k}}{1+\alpha_{m, k} \overline{\gamma_{m b, k}}}\right\}$ (= arg $\max _{k}\left\{\left.\frac{d r}{d \alpha_{b, k}}\right|_{\alpha_{b, k}=0}\right\}$ ). Recall that, due to conditions (C1) and (C2), we have that $\frac{d^{2} r}{d \alpha_{b, k}{ }^{2}}<0$, and therefore $\frac{d r}{d \alpha_{b, k}}$ is monotonically decreasing, $\forall k$. It follows that for any $\alpha_{b, k^{*}} \in[0,1]$ and any $k \in\{1, \ldots, K\}$, either there exists a (unique) $\alpha_{b, k} \in[0,1]$ such that $\frac{d r}{d \alpha_{b, k}}=\frac{d r}{d \alpha_{b, k^{*}}}$, or $\frac{d r}{d \alpha_{b, k}}<\frac{d r}{d \alpha_{b, k^{*}}}, \forall \alpha_{b, k} \in[0,1]$

Consider Algorithm 3 (SolveSubproblemb) and let $\left\{\alpha_{b, k}^{*}\right\}$ be the solution returned by the algorithm. Note that the binary search for finding $\alpha_{b, k^{*}}^{*}$ and for determining $\alpha_{b, k}^{*}$ 's in SolveSubproblemb is correct from the choice of $k^{*}$ and because $\frac{d r}{d \alpha_{b, k}}$ is monotonically decreasing, $\forall k$.

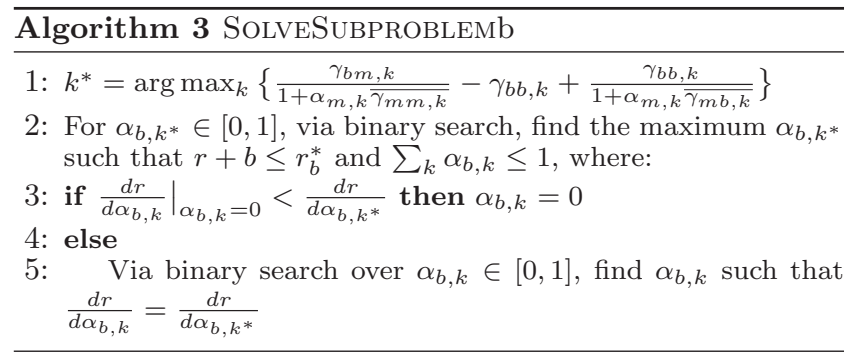

We first show that $\left\{\alpha_{b, k}^{*}\right\}$ is a local maximum for $\left(Q_{b}\right)$. Because of the algorithm's termination conditions, it must be either $\sum_{k} \alpha_{b, k}^{*}=1$ or $r_{b}=r_{b}^{*}$. If $\sum_{k} \alpha_{b, k}^{*}=1$, then to move to any alternative solution, the total change must be $\sum_{k} \Delta \alpha_{b, k} \leq 0$, or, equivalently $\Delta \alpha_{b, k^{*}} \leq-\sum_{k \neq k^{*}} \Delta \alpha_{b, k}$. As $\frac{d r}{d \alpha_{b, k}} \leq \frac{d r}{d \alpha_{b, k^{*}}}$, it follows that $\sum_{k} \frac{d r}{d \alpha_{b, k}} \Delta \alpha_{b, k} \leq 0$, which is the first-order optimality condition. Now suppose that $r_{b}=r_{b}^{*}$. Since $\frac{d r}{d \alpha_{b, k}}=\frac{d r_{b}}{d \alpha_{b, k}}+\frac{d r_{m}}{d \alpha_{b, k}}>0$ and $\frac{d r_{b}}{d \alpha_{b, k}}>$
$0, \frac{d r_{m}}{d \alpha_{b, k}}<0$, to keep the solution feasible (i.e., to keep $\left.r_{b} \leq r_{b}^{*}\right)$, we must have $\sum_{k} \frac{d r_{b, k}}{d \alpha_{b, k}} \Delta \alpha_{b, k} \leq 0$, which implies $\sum_{k} \frac{d r}{d \alpha_{b, k}} \Delta \alpha_{b, k} \leq 0$. Therefore, $\left\{\alpha_{b, k}^{*}\right\}$ computed by SolveSubproblemb is a local optimum.

In fact, for any local optimum: $\frac{d r}{d \alpha_{b, k}} \leq \frac{d r}{d \alpha_{b, k^{*}}}$, otherwise we can construct a better solution. Suppose that $\frac{d r}{d \alpha_{b, j}}>\frac{d r}{d \alpha_{b, k^{*}}}$ for some $j$. Then if $\frac{d r_{b}}{d \alpha_{b, j}} \leq \frac{d r_{b}}{d \alpha_{b, k^{*}}}$, we can choose a sufficiently small $\Delta>0$, so that the solution $\left\{\alpha_{b, k}^{\prime}\right\}$ with $\alpha_{b, j}^{\prime}=\alpha_{b, j}+\Delta, \alpha_{b, k^{*}}^{\prime}=\alpha_{b, k^{*}}-\Delta$, and $\alpha_{b, k}^{\prime}=\alpha_{b, k}$ for $k \notin\left\{j, k^{*}\right\}$ is feasible. For such a solution $\sum_{k} \frac{d r}{d \alpha_{b, k}}\left(\alpha_{b, k}^{\prime}-\right.$ $\left.\alpha_{b, k}\right)>0$, and therefore, it is not a local optimum. Conversely, if $\frac{d r_{b}}{d \alpha_{b, j}}>\frac{d r_{b}}{d \alpha_{b, k^{*}}}$, we can choose sufficiently small $\Delta_{1}, \Delta_{2}>0$ such that $\Delta_{2}>\Delta_{1}$ and $\frac{d r}{d \alpha_{b, j}} \Delta_{1}>\frac{d r}{d \alpha_{b, k^{*}}} \Delta_{2}$. Then, we can construct an $\left\{\alpha_{b, k}^{\prime}\right\}$ with $\alpha_{b, j}^{\prime}=\alpha_{b, j}+\Delta_{1}$, $\alpha_{b, k^{*}}^{\prime}=\alpha_{b, k^{*}}-\Delta_{2}$, and $\alpha_{b, k}^{\prime}=\alpha_{b, k}$ for $k \notin\left\{j, k^{*}\right\}$ that is feasible. Again, we have $\sum_{k} \frac{d r}{d \alpha_{b, k}}\left(\alpha_{b, k}^{\prime}-\alpha_{b, k}\right)>0$, and $\left\{\alpha_{b, k}\right\}$ cannot be a local maximum.

Finally, since $\left\{\alpha_{b, k}^{*}\right\}$ returned by SolveSubproblemb satisfies $\alpha_{b, k}^{*} \geq \alpha_{b, k}^{\prime}$ for any other local maximum $\left\{\alpha_{b, k}^{\prime}\right\}$ and the objective is strictly increasing in all $\alpha_{b, k}$ 's, $\left\{\alpha_{b, k}^{*}\right\}$ must be a global maximum. From the strict monotonicity of $\frac{d r}{d \alpha_{b, k}}$, this maximum is unique. The proof for $\left(Q_{R, m}\right)$ when $r_{b}^{*} \geq s_{b}$ uses similar arguments and is omitted.

\subsection{A Simple Power Allocation Heuristic}

Even though the algorithm described in the previous section will lead to the optimal or a near-optimal TDFD capacity region in many cases of interest, it may not be suitable for a real-time implementation. This motivates us to develop a simple heuristic that performs well in most cases and is based on the observations we made while implementing the algorithms described in previous sections.

The intuition for the heuristic is that around the points $\left(0, \overline{r_{m}}\right)$ and $\left(\overline{r_{b}}, 0\right)$, one of the two rates is very low, and the power allocation at the station with the high rate behaves as the optimal HD power allocation. When the SNR on each channel and at both stations is high compared to the XINR, the power allocation around the point $\left(s_{b}, s_{m}\right)$ has the shape of the power allocation in the high SINR approximation $^{4}$. When the SNR compared to the XINR is high on some channels, but not high on the other channels, then it may be better to use some of the channels with low SNR as HD. For practical implementations of compact FD transceivers, the channels with the higher XINR typically appear closer to the edges of the frequency band. The pseudocode of the heuristic for the case $r_{b}^{*} \leq s_{b}$ is provided in Algorithm 4 (PA-Heuristic) in the appendix. The pseudocode for the case $r_{b}^{*}>s_{b}$ is analogous to the $r_{b}^{*} \leq s_{b}$ case and is omitted. Here, $\left(s_{b}, s_{m}\right)$ is obtained as the rate pair that maximizes the sum rate under the high SINR approximation, as in [17].

For the FD capacity region determined by the heuristic, we further run a convex hull computation algorithm [9] to determine the FD + TDD capacity region. The total running time is $O\left(N K^{2} \log \left(\sum_{k} \overline{\gamma_{b b, k}} /(K \varepsilon)\right)\right)$ for computing $N$ points on the FD capacity region boundary by using PAHeuristic, plus additional $O(N)$ for convexifying the ca-

\footnotetext{
${ }^{4}$ See [17] for the high SINR approximation power allocation.
} 


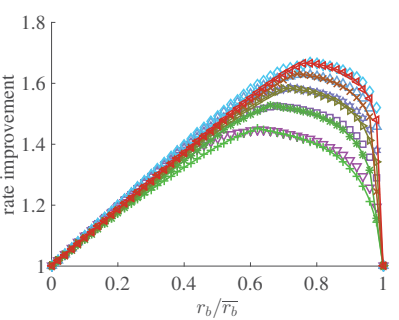

(a)

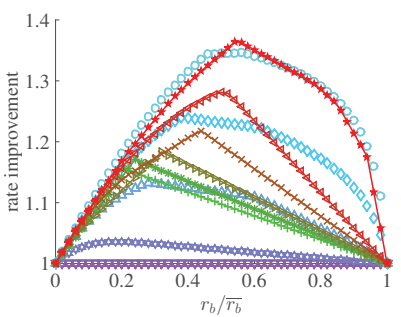

(d)

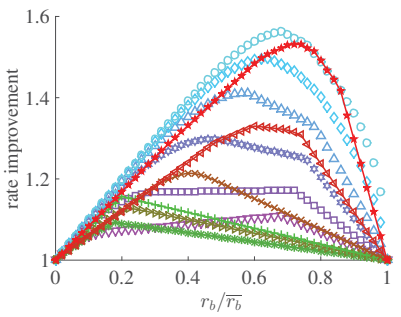

(g)

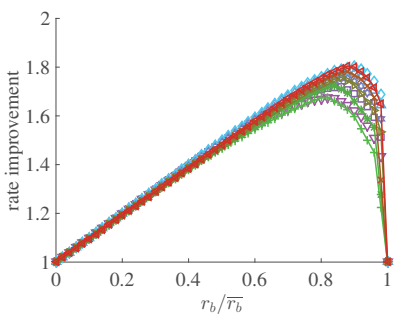

(b)

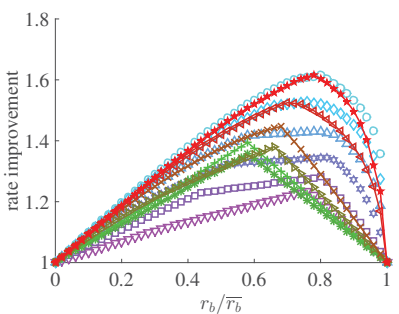

(e)

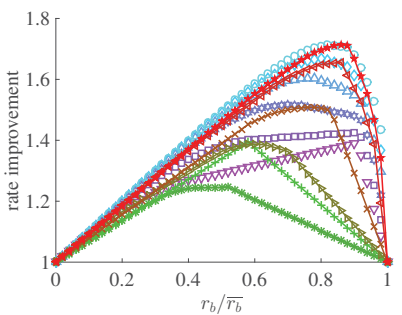

(h)

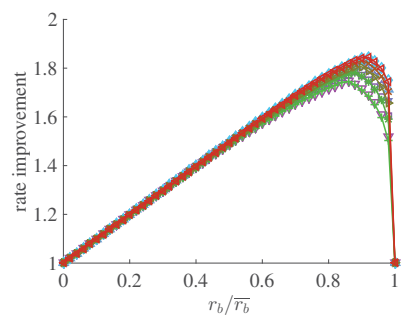

$-\overline{\gamma_{m b}}=\overline{\gamma_{b m}}=30 \mathrm{~dB}$, AltMax $\nabla \overline{\gamma_{m b}}=\overline{\gamma_{b m}}=30 \mathrm{~dB}$, Heuristic $* \overline{\gamma_{m b}}=\overline{\gamma_{b m}}=35 \mathrm{~dB}$, AltMax 口 $\overline{\gamma_{m b}}=\overline{\gamma_{b m}}=35 \mathrm{~dB}$, Heuristic $\triangleright \overline{\gamma_{m b}}=\overline{\gamma_{b m}}=40 \mathrm{~dB}$, AltMax \& $\overline{\gamma_{m b}}=\overline{\gamma_{b m}}=40 \mathrm{~dB}$, Heuristic $* \overline{\gamma_{m b}}=\overline{\gamma_{b m}}=45 \mathrm{~dB}$, AltMax $\Delta \overline{\gamma_{m b}}=\overline{\gamma_{b m}}=45 \mathrm{~dB}$, Heuristic $\varangle \overline{\gamma_{m b}}=\overline{\gamma_{b m}}=50 \mathrm{~dB}$, AltMax

(c)

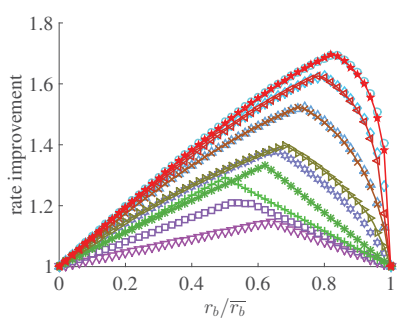

$+\overline{\gamma_{m b}}=\overline{\gamma_{b m}}=0 \mathrm{~dB}$, AltMax $\nabla \overline{\gamma_{m b}}=\overline{\gamma_{b m}}=0 \mathrm{~dB}$, Heuristic $* \overline{\gamma_{m b}}=\overline{\gamma_{b m}}=5 \mathrm{~dB}$, AltMax 口 $\overline{\gamma_{m b}}=\overline{\gamma_{b m}}=5 \mathrm{~dB}$, Heuristic $\rightarrow \overline{\gamma_{m b}}=\overline{\gamma_{b m}}=10 \mathrm{~dB}$, AltMax \# $\overline{\gamma_{m b}}=\overline{\gamma_{b m}}=10 \mathrm{~dB}$, Heuristic $* \overline{\gamma_{m b}}=\overline{\gamma_{b m}}=15 \mathrm{~dB}$, AltMax $\Delta \overline{\gamma_{m b}}=\overline{\gamma_{b m}}=15 \mathrm{~dB}$, Heuristic $\varangle \overline{\gamma_{m b}}=\overline{\gamma_{b m}}=20 \mathrm{~dB}$, AltMax $\diamond \overline{\gamma_{m b}}=\overline{\gamma_{b m}}=20 \mathrm{~dB}$, Heuristic $\star \overline{\gamma_{m b}}=\overline{\gamma_{b m}}=25 \mathrm{~dB}$, AltMax $\circ \overline{\gamma_{m b}}=\overline{\gamma_{b m}}=25 \mathrm{~dB}$, Heuristic

(f)

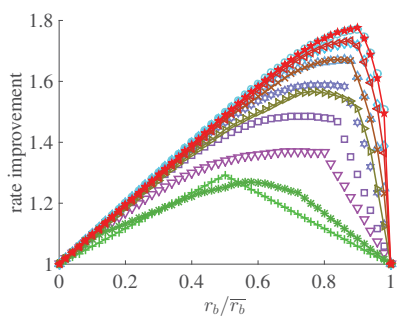

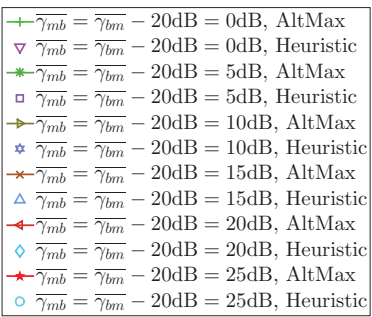

(i)

Figure 8: Rate improvements for $\overline{\gamma_{b b, k}}$ and $\overline{\gamma_{m m, k}}$ from Fig. 2. The leftmost column of graphs corresponds to $\overline{\gamma_{m m, k}}$ from Fig. 2(b), the middle column corresponds to $\overline{\gamma_{m m, k}}$ from Fig. 2(c), and the rightmost column corresponds to $\overline{\gamma_{m m, k}}$ from Fig. 2(d). $\overline{\gamma_{b b, k}}$ is selected according to Fig. 2(a). When rate improvements are at least $1.4 \times$, the heuristic performs similar to or better than the alternating maximization.

pacity region. Note that in practice $K$ and $N$ are at the order of 100, which makes this algorithm real-time.

The comparison of the rate improvement for FD + TDD operation determined by PA-HEURISTIC and the alternating maximization algorithm described in the previous section is shown in Fig. 8. The results shown in Fig. 8 were obtained assuming that $\overline{\gamma_{b m, 1}}=\overline{\gamma_{b m, K}} \ldots \equiv K \overline{\gamma_{b m}}, \overline{\gamma_{m b, 1}}=\ldots=$ $\overline{\gamma_{m b, K}} \equiv K \overline{\gamma_{m b}}$, and $\overline{\gamma_{m m, k}}, \overline{\gamma_{b b, k}}$ from Fig. 2. The alternating maximization algorithm can provide an optimal solution only when conditions (C1) and (C2) are non-restrictive, i.e., when $\overline{\gamma_{b m, k}} \geq \overline{\gamma_{b b, k}}\left(1+\overline{\gamma_{m m, k}}\right)$ and $\overline{\gamma_{m b, k}} \geq \overline{\gamma_{m m, k}}\left(1+\overline{\gamma_{b b, k}}\right)$, $\forall k$. For $\overline{\gamma_{b b, k}}$ from Fig. 2(a) and $\overline{\gamma_{m m, k}}$ from Fig. 2(b), (c), and $(\mathrm{d}),(\mathrm{C} 1)$ and $(\mathrm{C} 2)$ are non-restrictive when (i) $\overline{\gamma_{b m}} \geq$ $39.1 \mathrm{~dB}, \overline{\gamma_{m b}} \geq 39.2 \mathrm{~dB}$, (ii) $\overline{\gamma_{b m}} \geq 32.8 \mathrm{~dB}, \overline{\gamma_{m b}} \geq 32.3 \mathrm{~dB}$, and (iii) $\overline{\gamma_{b m}} \geq 25.3 \mathrm{~dB}, \overline{\gamma_{m b}} \geq 25.3 \mathrm{~dB}$, respectively.

As Fig. 8(a)-(c) shows, when $(\mathrm{C} 1)$ and $(\mathrm{C} 2)$ are nonrestrictive, the alternating maximization algorithm and the PA-HeuRISTIC provide almost identical results (minor differences are mainly due to a numerical error in computation). Moreover, when the smallest upper bound on $\alpha_{b, k}$ 's and $\alpha_{m, k}$ 's imposed by (C1) and (C2) is no higher than $5 / K$, i.e., for (i) $\overline{\gamma_{b m}} \geq 28.9 \mathrm{~dB}, \overline{\gamma_{m b}} \geq 29.7 \mathrm{~dB}$, (ii) $\overline{\gamma_{b m}} \geq 22.6 \mathrm{~dB}$, $\overline{\gamma_{m b}} \geq 23.4 \mathrm{~dB}$, and (iii) $\overline{\gamma_{b m}} \geq 15.2 \mathrm{~dB}, \overline{\gamma_{m b}} \geq 15.9 \mathrm{~dB}$, for $\overline{\gamma_{m m, k}}$ from Fig. 2(b), (c), and (d), respectively, the differences between the alternating maximization algorithm and the PA-Heuristic are still negligible (Fig. 8(a)-(i)).

When (C1) and (C2) are restrictive (Fig. 8(d)-(i)), all fol- lowing cases may happen: (i) the alternating maximization outperforms the PA-Heuristic, (ii) the PA-HeuRistic outperforms the alternating maximization, and (iii) both have similar performance. Case (i) typically happens when most channels are allocated as HD by the alternating maximization, with some of them allocated to the BS, and others to the MS. In this case the rate improvements predominantly come from using higher total irradiated power compared to TDD, rather than from using full-duplex. Note that the PA-Heuristic allows the HD channels to be assigned either only to the BS or only to the MS, but not both. Case (ii) happens when $(\mathrm{C} 1)$ and $(\mathrm{C} 2)$ restrict the part of the feasible region where high rate improvements are possible; namely, when either both $\overline{\gamma_{b m}}$ and $\overline{\gamma_{m b}}$ are low, or when $\overline{\gamma_{b m}}$ is much $(20 \mathrm{~dB})$ higher than $\overline{\gamma_{m b}}$.

\section{CONCLUSION}

We presented a theoretical study of the capacity region of FD in both the single and multi-channel cases. We developed algorithms that not only allow characterizing the region but can also be used for asymmetrical rate allocation. We numerically demonstrated the gains from FD.

While significant attention has been given to resource allocation in HD OFDM networks (e.g., [12] and references therein), as we demonstrated, the special characteristics of FD pose many new challenges. In particular, the design of 
MAC protocols that support the co-existence of HD and FD users while providing fairness is an open problem. Moreover, there is a need for experimental evaluation of scheduling, power control, and channel allocation algorithms tailored for the special characteristics of FD.

\section{ACKNOWLEDGEMENTS}

This work was supported in part by the NSF grant ECCS1547406, the Qualcomm Innovation Fellowship, and the People Programme (Marie Curie Actions) of the European Union's Seventh Framework Programme (FP7/2007-2013) under REA grant agreement $\mathrm{n}^{\mathrm{o}}[$ PIIF-GA-2013-629740].11. We thank Jin Zhou and Harish Krishnaswamy for providing us with the cancellation data from [24].

\section{REFERENCES}

[1] E. Ahmed, A. M. Eltawil, and A. Sabharwal. Rate gain region and design tradeoffs for full-duplex wireless communications. IEEE Trans. Wireless Commun., 12(7):3556-3565, 2013.

[2] E. Aryafar, M. A. Khojastepour, K. Sundaresan, S. Rangarajan, and M. Chiang. MIDU: enabling MIMO full duplex. In Proc. ACM MobiCom'12, 2012.

[3] J. Bai and A. Sabharwal. Distributed full-duplex via wireless side-channels: Bounds and protocols. IEEE Trans. Wireless Commun., 12(8):4162-4173, 2013.

[4] A. Beck. On the convergence of alternating minimization for convex programming with applications to iteratively reweighted least squares and decomposition schemes. SIAM J. Optimiz., 25(1):185-209, 2015.

[5] D. Bharadia, E. McMilin, and S. Katti. Full duplex radios. In Proc. ACM SIGCOMM'13, 2013.

[6] W. Bi, X. Su, L. Xiao, and S. Zhou. On rate region analysis of full-duplex cellular system with inter-user interference cancellation. In Proc. IEEE ICCW'15, 2015.

[7] W. Cheng, X. Zhang, and H. Zhang. Optimal dynamic power control for full-duplex bidirectional-channel based wireless networks. In Proc. IEEE INFOCOM'13, 2013.

[8] J. Choi, M. Jain, K. Srinivasan, P. Levis, and S. Katti. Achieving single channel, full duplex wireless communication. In Proc. ACM MobiCom'10, 2010.

[9] T. H. Cormen. Introduction to algorithms. MIT press, 2009.

[10] M. Duarte, C. Dick, and A. Sabharwal. Experiment-driven characterization of full-duplex wireless systems. IEEE Trans. Wireless Commun., 11(12):4296-4307, 2012.

[11] J. Gorski, F. Pfeuffer, and K. Klamroth. Biconvex sets and optimization with biconvex functions: a survey and extensions. Math. Method. Oper. Res., 66(3):373-407, 2007.

[12] J. Huang, V. G. Subramanian, R. Agrawal, and R. A. Berry. Downlink scheduling and resource allocation for OFDM systems. IEEE Trans. Wireless Commun., 8(1):288-296, Jan. 2009.

[13] M. Jain, J. Choi, T. Kim, D. Bharadia, S. Seth, K. Srinivasan, P. Levis, S. Katti, and P. Sinha. Practical, real-time, full duplex wireless. In Proc. ACM MobiCom'11, 2011.

[14] M. Khojastepour, K. Sundaresan, S. Rangarajan, X. Zhang, and S. Barghi. The case for antenna cancellation for scalable full-duplex wireless communications. In Proc. ACM HotNets'11, 2011.

[15] D. Korpi, T. Riihonen, and M. Valkama. Achievable rate regions and self-interference channel estimation in hybrid full-duplex/half-duplex radio links. In Proc. IEEE CISS, 2015.

[16] W. Li, J. Lilleberg, and K. Rikkinen. On rate region analysis of half-and full-duplex OFDM communication links. IEEE J. Sel. Areas Commun., 32(9):1688-1698, Sept. 2014.
[17] J. Marašević, J. Zhou, H. Krishnaswamy, Y. Zhong, and G. Zussman. Resource allocation and rate gains in practical full-duplex systems. In Proc. ACM SIGMETRICS'15, 2015.

[18] J. Marašević and G. Zussman. On the capacity regions of single-channel and multi-channel full-duplex links, May 2016. arXiv preprint, http://arxiv.org/abs/1605.07559.

[19] J. M. Ortega and W. C. Rheinboldt. Iterative solution of nonlinear equations in several variables. Academic Press, 1970.

[20] A. Sabharwal, P. Schniter, D. Guo, D. Bliss, S. Rangarajan, and R. Wichman. In-band full-duplex wireless: Challenges and opportunities. IEEE J. Sel. Areas Commun. 32(9):1637-1652, Sept. 2014.

[21] X. Xie and X. Zhang. Does full-duplex double the capacity of wireless networks? In Proc. IEEE INFOCOM'14, 2014.

[22] Y. Yang and N. B. Shroff. Scheduling in wireless networks with full-duplex cut-through transmission. In Proc. IEEE INFOCOM'15, 2015.

[23] G. Zheng. Joint beamforming optimization and power control for full-duplex MIMO two-way relay channel. IEEE Trans. Signal Process., 63(3):555-566, 2015.

[24] J. Zhou, T.-H. Chuang, T. Dinc, and H. Krishnaswamy. A receiver with reconfigurable self-interference cancellation based on RF frequency-domain equalization supporting $>20 \mathrm{MHz}$ cancellation bandwidth for FDD, co-existence and same-channel full duplex applications. In Proc. IEEE ISSCC'15, 2015.

\section{APPENDIX}

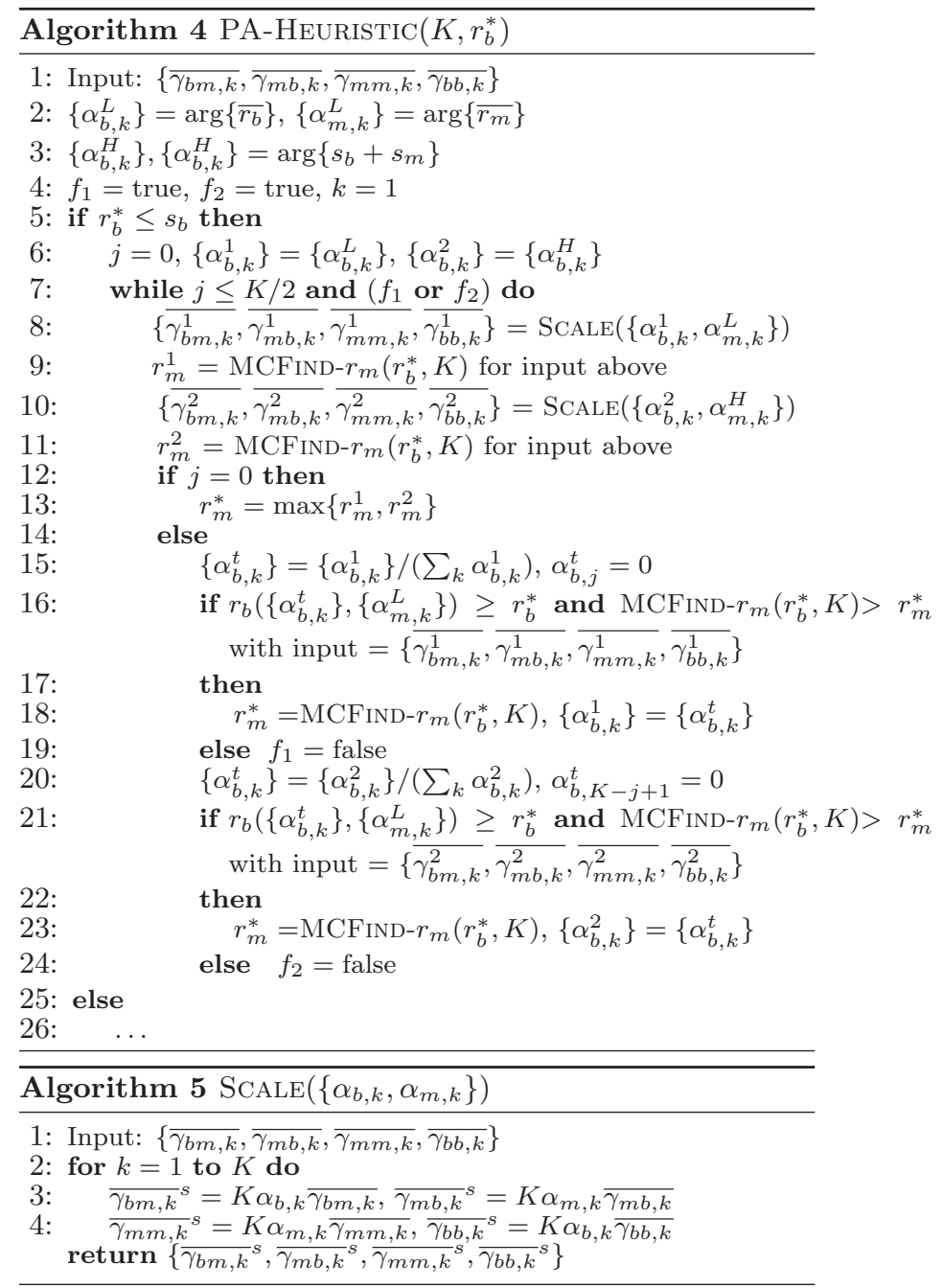

